\title{
Minimalistic Models of an Energy Efficient Vertical Hopping Robot
}

\author{
Xiaoxiang Yu and Fumiya Iida, Member, IEEE,
}

\begin{abstract}
The use of free vibration in elastic structure can lead to energy efficient robot locomotion, since it significantly reduces the energy expenditure if properly designed and controlled. However, it is not well understood how to harness the dynamics of free vibration for the robot locomotion, because of the complex dynamics originated in discrete events and energy dissipation during locomotion. From this perspective, the goal of this paper is to propose a design strategy of hopping robot based on elastic curved beams and actuated rotating masses, and identify the minimalistic model that can characterize the basic principle of robot locomotion. Since the robot mainly exhibits vertical hopping, three one-dimensional models are examined that contain different configurations of simple spring-damper-mass components. The real-world and simulation experiments show that one of the models best characterizes the robot hopping, through analyzing the basic kinematics and negative works in actuation. Based on this model, the self-stability of hopping motion under disturbances is investigated and design and control parameters are analyzed for the energy efficient hopping. Additionally, further analyses show that this robot can achieve the energy efficient hopping with the variation in payload, and the source of energy dissipation of the robot hopping is investigated.
\end{abstract}

Index Terms-legged locomotion, energy efficiency, motion control, robot dynamics, modeling.

\section{INTRODUCTION}

$\mathbf{L}$ EGGED robot locomotion has been attracting an increasing interest because of the needs of behavioral dexterity and diversity in mobile robot systems. Previously a number of legged robot platforms were proposed (e.g. [3]-[5]), and a variety of techniques were reported to synthesize robust gait patterns to deal with different types of environments [6], [7]. It is, however, still largely unknown how to solve the problems related to energy efficiency of legged robot locomotion even though they have been known as one of the most critical challenges for a long time [8]-[12].

One of the most energy efficient legged robots is a passive dynamic walker, which can achieve bipedal locomotion comparable to human walking on a shallow slope without active

Manuscript received October 6, 2012; Revised December 12, 2012 and March 19, 2013; Accepted for publication May 11, 2013. This study was supported by the Swiss National Science Foundation Grant No. PP00P2123387/1, and the Swiss National Science Foundation through the National Centre of Competence in Research Robotics. This paper was presented in part in [1] and [2].

$\mathrm{X}$. Yu is with Bio-Inspired Robotics Lab, Institute of Robotics and Intelligent Systems, ETH Zurich, Leonhardstrasse 27, 8092 Zurich, Switzerland and Artificial Intelligence Laboratory, University of Zurich, Switzerland (email: xiaoxiang.yu@mavt.ethz.ch).

F. Iida is with Bio-Inspired Robotics Lab, Institute of Robotics and Intelligent Systems, ETH Zurich, Switzerland (email: fumiya.iida@mavt.ethz.ch).

Copyright (c) 2009 IEEE. Personal use of this material is permitted. However, permission to use this material for any other purposes must be obtained from the IEEE by sending a request to pubs-permissions@ieee.org control or energy input [13]. Based on the passive dynamics, they can also walk on the level ground with small active power sources, and it is reported that their energy efficiency can be comparable to that of humans [14], [15]. However these robots require well-defined environment and cannot recover from large disturbances because of the lack of basic stability.

To account for some of the problems in the passive dynamic walkers, many researchers investigated compliant elements in the robot structure, because the compliant dynamics can be used to recycle kinetic energy during locomotion without losing it by impact or deceleration [11], [16]-[18]. The main body of previous literature in this line of research focused on the so-called spring-mass model, which consists of a point mass and massless spring [19], [20], and they identified that the model can explain some of the underlying mechanical dynamics and its locomotion stability in animal locomotion [21], [22]. Furthermore, inspired from the research on the spring-mass models, a number of researchers investigated how to reduce energy dissipation during legged locomotion while maintaining stability. As a result, it was reported that the robots based on these approaches were able to run and hop at a level of energy efficiency comparable to that of human running [23], [24]. In addition, a significantly simpler approach to develop energetically efficient hopping robots was also proposed, in which forced oscillation of an elastic curved beam is used as the main drive of hopping robot locomotion [1], [25]-[27]. It turns out that the use of elastic curved beams in hopping robots is beneficial not only to simplify the manufacturing processes, but also to reduce energetic cost of locomotion due to the light-weight leg structures and relatively small requirement in actuation. Although these demonstrations in the past are convincing, the underlying principles are still largely unknown to substantially increase the energy efficiency of robot locomotion, and one of the most significant challenges seems to be the identification of simple locomotion models that can be used for the systematic exploration of the complex problem of energy efficiency robot locomotion.

From this perspective, the goal of this paper is to develop a minimalistic model that can be used, on the one hand, for systematic design of energetically efficient hopping robots, and on the other, for explaining the basic underlying principles. As it becomes clearer later in this paper, the analysis of energy efficiency is not trivial even in a simple vertical hopping robot because there are many mechanical and control parameters involved in the dynamics. Therefore, in this paper, we start the investigation of hopping behaviors in a simple real-world robot and its model (i.e. a simple spring-mass-damper system), and gradually increase the complexity by including additional 
TABLE I: Parameters of robot

\begin{tabular}{|c|c|}
\hline Parameter & Value \\
\hline height of robot & $0.45 \mathrm{~m}$ \\
\hline width of base & $0.3 \mathrm{~m}$ \\
\hline length of base & $0.25 \mathrm{~m}$ \\
\hline radius of rotating mass & $0.145 \mathrm{~m}$ \\
\hline maximum hopping height & $0.02 \mathrm{~m}$ \\
\hline mass of base & $0.06 \mathrm{~kg}$ \\
\hline mass of beam & $0.1 \mathrm{~kg}$ \\
\hline mass of payload & $0.5 \mathrm{~kg}$ \\
\hline mass of DC motor & $0.1 \mathrm{~kg}$ \\
\hline mass of rotating mass & $0.03 \mathrm{~kg}$ \\
\hline stiffness constant of beam along the vertical direction & $300 \mathrm{~N} / \mathrm{m}$ \\
\hline damping coefficient of beam & $1.2 \mathrm{Ns} / \mathrm{m}$ \\
\hline angular velocity of rotating mass & $17.85 \mathrm{rad} / \mathrm{s}$ \\
\hline average input power of robot & $1.44 \mathrm{~W}$ \\
\hline
\end{tabular}

masses and spring-damper elements until behaviors of the model match to the real-world robots. In addition, based on the identified minimalistic model, we also investigate the underlying principles and scalability of the proposed approach.

The rest of this paper is structured as follows. In Section II, we describe the target robot platform and its basic behavioral characteristics. Section III presents the models we investigate in this paper as well as the system identification of them. In Section IV, we explain a comprehensive analysis of models, and presents their feasibility. And finally, in Section V, we conclude the papers by discussing the implications and future work of this project.

\section{VERTICAL HOPPING RobOT}

In this paper, we introduce a curved beam robot that was designed to investigate energy efficient robot locomotion. This section first describes the design of physical experimental platform, and then introduce the method of experiment to systematically investigate energy efficiency in robot locomotion.

\section{A. Structure of Robot}

As shown in Fig.1a, the robot consists of a body made by an elastic curved beam, a foot base, a payload, and an actuator. The curved beam is made of aluminum and structured into a Cshape, which was found to provide a stable hopping behavior [25]. The foot base is also made of aluminum and has a $\mathrm{H}$ shape structure to provide basic stability during locomotion. And we employed a DC motor (Maxon RE $\varnothing 2525 \mathrm{~mm}, 10 \mathrm{~W}$ ) without any gearbox to maximize the actuation efficiency. At both ends of the drive shaft of the motor, two rotating masses are attached which provide energy into the whole system for locomotion. The parameters of the robot are given in Table I.

When connected to the power, the DC motor drives the rotating masses around the shaft with approximately constant speed and this rotation induces centrifugal force. By increasing angular velocity of these rotating masses, the whole system oscillates with larger amplitude, and at a frequency close to resonance, the base takes off from the ground and the robot starts hopping. If the curved beam is structured into a certain shape, this hopping behavior occurs mainly along the vertical direction as shown in Fig. 1(b), which is the target behavior we investigate in this paper.

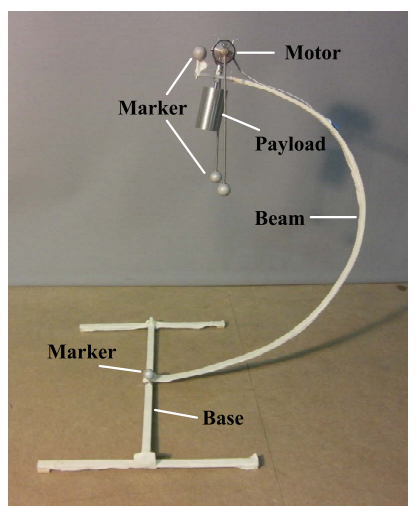

(a)
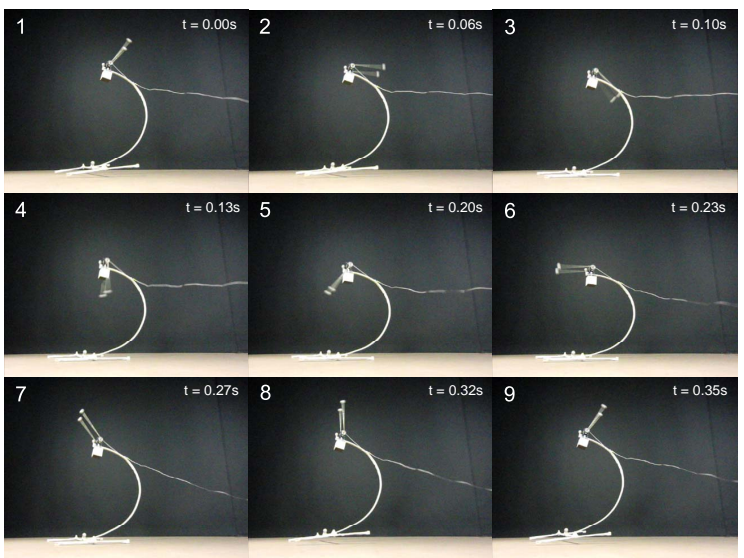

(b)

Fig. 1: (a) The curved beam robot. (b) Time series photographs for one cycle stable vertical hopping of the curved beam robot. The time is shown at the upper right of each snapshot. The upper left numbers index the order. The rotation direction of the motor is clockwise. The snapshots 3 and 7 show the moments when the robot touches down and takes off the ground. The snapshot 8 shows the moment when the robot reaches the maximum hopping height.

\section{B. Experimental Method}

By using the robot platform in Fig. 1(a), a series of hopping experiments were conducted. An indoor flat wooden floor was used as the ground for the robot hopping. A power supply provided a constant voltage that was sufficient to drive the motor on the robot and the power was measured and recorded. In addition, a commercial motion capture system OptiTrack was employed to track the 3D trajectories of the robot motion. Its measurement accuracy is less than $1 \mathrm{~mm}$ and the tracking speed is 120 frames per second. As shown in Fig. 1(a), four markers are attached on the base, top end of the beam and both ends of the rotating masses of the robot. By using these markers, we were able to record the robot kinematics in a $3 \mathrm{D}$ coordinate system real-time during the experiments.

It is important to note that the robot shown in Fig. 1(a) is able to hop forward or backward by inducing the free vibration [25]. However, in order to simplify the analysis of the dynamics, in this paper, we only focus on the vertical hopping by minimizing horizontal motions. The vertical hopping of 
this robot can be induced by adjusting the attachment angle of elastic curved beam to the foot base without disturbing the basic hopping dynamics too much. Therefore, by setting the attachment angle such that the primary axis of beam oscillation be upright, the robot hops in a vertical direction only, hence we are able to analyze behavioral characteristics by using the one-dimensional spring-damper-mass models we explain in the next section.

\section{Energy Efficiency}

For a systematic evaluation, here we define a quantification method of hopping behavior, the so-called Cost of Hopping $(\mathrm{CoH})$, which can be defined as:

$$
C o H=\frac{E}{M_{\text {total }} g h},
$$

where $E$ denotes the mechanical energy dissipation in one cycle of stable hopping behavior, while $M_{\text {total }}, g$ and $h$ denote the total mass of the robot, gravity acceleration and maximum hopping height, respectively. Thus the goal of energy efficient vertical hopping is to reduce $\mathrm{CoH}$ (i.e. reduce $E$ while increasing $M_{\text {total }}$ and $h$ ).

From the parameters in Table I, $\mathrm{CoH}$ of the robot shown in Fig. 1(a) can be calculated as

$$
\begin{aligned}
\mathrm{CoH} & =\frac{1.44 \times \frac{2 \pi}{17.85}}{(0.06+0.1+0.5+0.1+0.03) \times 9.81 \times 0.02}, \\
& \approx 3.27 .
\end{aligned}
$$

The efficiency of our hopping robot can be further improved as low as 0.19 which will be shown in Section IV.B. With this quantification, the hopping efficiency of our robot can be compared to the other systems. For example, according to [28], our robot seems to be as efficient as human jumping because the energy efficiency was reported as 3.28. It is, however, important to note that, $\mathrm{CoH}$ is calculated by absolute hopping hight, thus a system with a larger leg length has an obvious advantage. In this sense, we could argue that the hopping efficiency of our robot surpassed that of human jumping because the leg length of our robot is $0.45 \mathrm{~m}$ as compared to the leg length of human subject which is $0.9 \mathrm{~m}$.

In addition, it is also possible to compare the efficiency of our robot to the other robots in a variety of sizes. The miniature jumping robot, for example, has a leg length of $0.05 \mathrm{~m}$ and $\mathrm{CoH}=12$ [29], and the ARL Monopod I with the leg length of $0.7 \mathrm{~m}$ has a $\mathrm{CoH}=1.36$ [30]. From this comparison, it can be said that our robot is one of the most energy efficient hopping robots, because it can still increase efficiency by optimized size, mass, and motor control as it becomes clearer later in this paper.

\section{SYSTEM IDENTIFICATION}

In order to explore the energy efficient vertical hopping, this section proposes three minimalistic models for the curved beam robot. Based on these models, the self-stability and hopping behavior of the robot are explored.

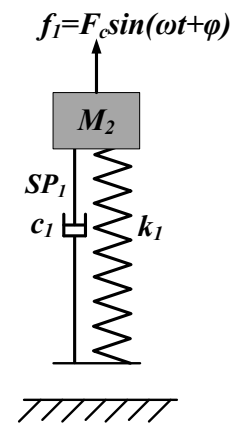

(a) Model I

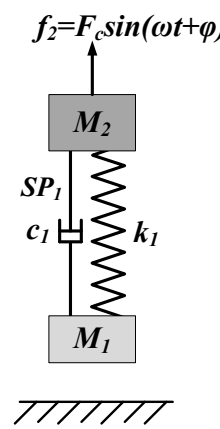

(b) Model II

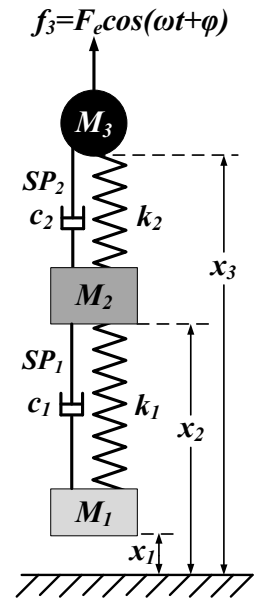

(c) Model III
Fig. 2: Three proposed models of the robot, i.e. Model I, II and III, which we investigate in this paper. Model I is the simplest one which contains a mass $M_{2}$, a massless springdamper $S P_{1}$, and an external force $f_{1}$. This model is then enhanced, in Model II, by including an additional mass $M_{1}$. Finally, in Model III, we consider an additional mass $M_{3}$ together with another spring-damper $S P_{2}$, which represents the actuated rotating mass in the robot. Six state variables are required to explain dynamics of Model III, which are the positions of three masses $x_{1}, x_{2}$ and $x_{3}$, and the velocities of them.

\section{A. Assumption of Models}

Based on the previous studies on the spring-mass models [19]-[22], three minimalistic models are systematically proposed in order to explore the dynamics of hopping robot, which are called Model I, II and III as shown in Fig. 2(a), 2(b) and 2(c). For the sake of simplicity, only vertical motions of these models are investigated while analyzing the effects of mass distributions, damping, ground impact and actuation dynamics.

Model I is the simplest spring-mass model, in which the payload and motor are represented by the point mass $M_{2}$ and the beam body is abstracted into a vertical massless linear spring $S P_{1}$ with stiffness $k_{1}$, viscous damping $c_{1}$ and natural length $L_{10}$. The mass of beam is assumed in $M_{2}$. Regarding the actuation model, the top of beam is mainly driven by a continuous centrifugal force induced by the rotating mass $M_{3}$ with a radius of $r$. Therefore, we can model the force induced by the rotatory part of system exerted on $M_{2}$ as an input force of Model I. Assuming the angular velocity of rotating mass is constant and its gravity is negligible, the input force is considered as a constant rotatory force. As shown in Fig. 3, the vertical component of such force is assumed to be sinusoidal as follows:

$$
f_{1}=F_{c} \sin (\omega t+\varphi),
$$

where $\omega$ is the angular velocity of rotating mass, and $F_{c}$ denotes the amplitude of input force and $\varphi$ is the angle between the rotating mass with respect to the horizon. Then, 


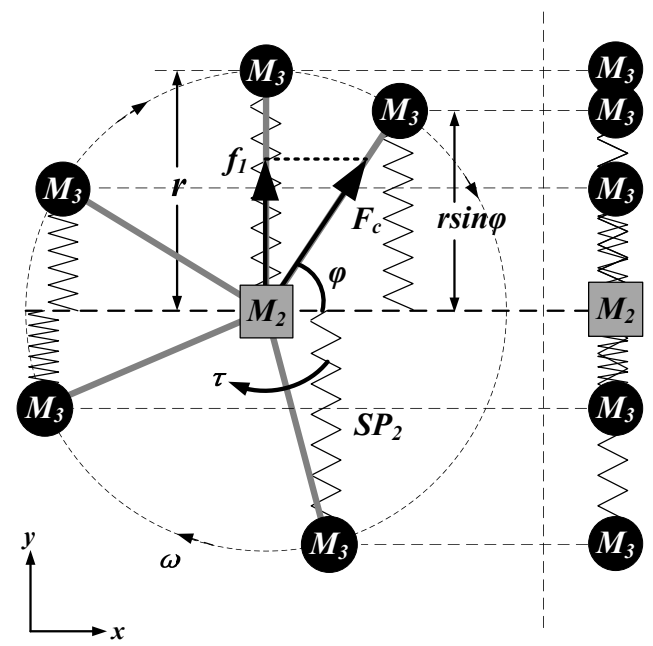

Fig. 3: The rotation of $M_{3}$ around $M_{2}$ can be considered as a vertical vibration around $M_{2}$, A linear spring $S P_{2}$ is assumed to connect $M_{3}$ and $M_{2}$ in order to model such a behavior.

$F_{c}$ can be approximated as the centrifugal force of $M_{3}$ :

$$
F_{c}=M_{3} \omega^{2} r \text {. }
$$

Note that, although the model in Fig. 2(a) does not indicate $M_{3}$, the actuation force $f_{1}$ considers to be equivalent to the centrifugal force induced by $M_{3}$, which is the reason it appears in (3).

In Model II, another point mass $M_{1}$ is included, which is attached to the low end of $S P_{1}$ to represent the base mass. Model II also employed the same assumption of the actuation in Model I, i.e. a sinusoidal force input is applied as follows:

$$
f_{2}=F_{c} \sin (\omega t+\varphi) .
$$

Again, here we consider the amplitude of this oscillation $F_{c}$ equivalent to the centrifugal force induced by $M_{3}$, which is shown in (3).

In Model III, an additional point mass $M_{3}$ is included to represent a rotating mass. As shown in Fig. 3, the projection of the rotating mass $M_{3}$ on the vertical direction can be explained as a vertical vibration around $M_{2}$. Considering the force between $M_{2}$ and $M_{3}$, their relative displacementforce response is consistent to the one-dimensional massspring system motion. Therefore, a massless linear spring $\mathrm{SP}_{2}$ is assumed to connect $M_{2}$ and $M_{3} . k_{2}$ and $c_{2}$ denote the stiffness and viscous damper, and the natural length is $L_{20}$. Consequently, the spring constant $k_{2}$ can be approximated as follows:

$$
k_{2}=\frac{F_{c}}{r}=M_{3} \omega^{2} .
$$

Here the relationship between $k_{2}$ and $M_{3}$ is derived from the fact that the maximum force generated by $S P_{2}$ has to match to the projection of centrifugal force of $M_{3}$ in the real-world robot onto the vertical plane. In addition, the natural length of $S P_{2}$ is assumed to be $L_{20}=0$, and the energy consumed inside the motor $E_{\text {motor }}$ is modeled as equaled to the one consumed on the damping of $S P_{2}$ with the coefficient $c_{2}$. Thus, the value of $c_{2}$ is dependent on $E_{\text {motor }}$. Note that, due to

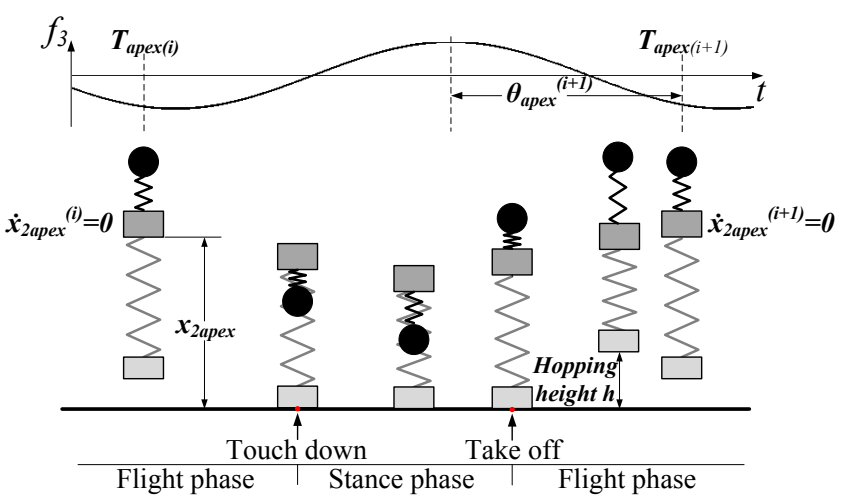

Fig. 4: One cycle behavior of Model III. The whole hopping motion has two phases: flight and stance. The behavior is following the sequence of apex point, touch down, lowest point, take off and apex point.

the physical limitation, it is hard to accurately measure $E_{\text {motor }}$ in the real robot so that $c_{2}$ cannot determined by the physical measurement.

The input force of the actuator in Model III was formulated as

$$
f_{3}=F_{e} \cos (\omega t+\varphi),
$$

where $F_{e}$ is the force from the motor exerted on $M_{3}$.

To estimate a reasonable $F_{e}$, we consider the force exerted on the rotating mass $M_{3}$ through the small torque generated by the motor. In the real-world robot experiments, we found that the torque output $\tau_{\exp }$ and power $P_{\exp }$ is very small (approximately about $0.08 \mathrm{Ns}$ and $1.5 \mathrm{~W}$ ) and the fluctuation is almost negligible, thus we employed the following formula to estimate $F_{e}$ :

$$
F_{e}=\frac{\tau}{r}=\frac{P_{e x p}}{\omega r}
$$

\section{B. Dynamics of Models}

Based on the assumptions, this subsection discusses the behaviors of models and investigates the dynamics. In the behaviors of Model I, II and III, $x_{1}, x_{2}, x_{3}, \dot{x_{1}}, \dot{x_{2}}, \dot{x_{3}}$ and $\theta$ are the state variables of system, where $x_{1}(t), x_{2}(t)$ and $x_{3}(t)$ denote the height of $M_{1}, M_{2}$ and $M_{3}$ with respect to the reference ground, while $\dot{x_{1}}(t), \dot{x_{2}}(t)$ and $\dot{x_{3}}(t)$ denote the upward velocity. $\theta(t)$, i.e. $\theta(t)=(\omega t+\varphi \bmod \pi)$, denotes the phase of sinusoidal input force at time $t$. Thus the initial condition variables of the system include $x_{1}(0), x_{2}(0), x_{3}(0)$, $\dot{x_{1}}(0), \dot{x_{2}}(0), \dot{x_{3}}(0)$ and $\theta(0)$. Furthermore, the initial phase of control sinusoid $\theta(0)$ is actually determined by $\varphi$. $\varphi$ is thus used for an initial condition variable of system.

Fig. 4 shows one cycle hopping behavior of Model III. Considering the similarities to Model III, the behaviors of Model I and II are not shown. One cycle of behavior includes two phases: stance phase and flight phase (i.e. $M_{1}$ contacts the ground or not). $f_{3}$, the unique actuation force of the system, induces the vibrations of $M_{1}, M_{2}$ and $M_{3}$. The moment when $M_{2}$ reaches the apex point is defined as the starting time $T_{\text {apex }}$. At $T_{\text {apex }}$, the displacement of $M_{2}$ respect to the ground is 
defined as $x_{2 a p e x}$ and the velocity $\dot{x}_{2 a p e x}=0$. The phase of $f$ at $T_{\text {apex }}$ is $\theta_{\text {apex }}$. During the cycle $i$ of the behavior, the system is in the flight phase at $T_{\text {apex }}^{i}$. After certain time, $M_{3}$ touches down the ground and the system starts the stance phase. When $M_{3}$ takes off from the ground, the system goes back to the flight phase. With the displacement rises, $M_{3}$ reaches the maximum height $h$, which is the hopping height of the system. In the end of cycle $i, M_{2}$ reaches the apex point again at $T_{\text {apex }}^{i+1}$.

To model the transition from a flight to a stance phase, the velocity of base mass is immediately set into zero when the collision happens. It implies that when the base mass with the velocity of $\dot{x_{1}}\left(T^{-}\right)$collides the ground at $T, \dot{x_{1}}$ is set to 0 at $T^{+}$, and $\dot{x_{1}}$ will stay at the zero until the dynamics enters the flight phase. Here, $T^{-}$and $T^{+}$denote the moments before and after touching down. Note that, Model I collides the ground without energy loss, since its base mass is zero. During the stance phase, when the spring force of $S P_{1}$ is equal to the gravity of $M_{1}$, the dynamics transitions from the stance phase to flight phase and $\dot{x_{1}}$ starts to accelerate. In Model I, the transition occurs when the spring reaches the natural length $L_{10}$.

This robot hopping behavior shows a typical discrete hybrid dynamics, which includes the stance phase and stance phase. We define $Q$ as the set of discrete phases of [flight, stance] and $q$ as the current phase. Then the discrete transition is denoted as

$$
\varphi\left(q, x_{1}\right)=\left\{\begin{array}{l}
\text { flight }, q=\text { flight } \text { and } x_{1}>0 \\
\text { stance }, q=\text { flight } \text { and } x_{1}=0 \\
\text { flight }, q=\text { stance } \text { and } x_{1}>0 \\
\text { stance, } q=\text { stance } \text { and } x_{1}=0,
\end{array}\right.
$$

where $x_{1}$ represents the coordinate of the spring bottom point in Model I. The motions of the continuous state variables are defined by the equations of motion.

Define $G_{1}, G_{2}$ and $G_{3}$ as the gravities of masses, and $f_{s p 1}$ and $f_{s p 2}$ as the spring forces of $S P_{1}$ and $S P_{2}$, where the compression forces are defined as positive. Then the equations of motion of three models are as follows.

Flight phase of Model I:

$$
M_{2} \ddot{x}_{2}=-G_{2}+f_{1} \text {. }
$$

Stance phase of Model I:

$$
M_{2} \ddot{x}_{2}=-G_{2}-f_{s p 1}+f_{1} \text {. }
$$

Flight phase of Model II:

$$
\left\{\begin{array}{l}
M_{1} \ddot{x}_{1}=-G_{1}+f_{s p 1} \\
M_{2} \ddot{x}_{2}=-G_{2}-f_{s p 1}+f_{2} .
\end{array}\right.
$$

Stance phase of Model II:

$$
\left\{\begin{aligned}
\ddot{x}_{1} & =0 \\
M_{2} \ddot{x}_{2} & =-G_{2}-f_{s p 1}+f_{2} .
\end{aligned}\right.
$$

Flight phase of Model III:

$$
\left\{\begin{array}{l}
M_{1} \ddot{x}_{1}=-G_{1}+f_{s p 1} \\
M_{2} \ddot{x}_{2}=-G_{2}-f_{s p 1}+f_{s p 2} \\
M_{3} \ddot{x}_{3}=-G_{3}-f_{s p 2}+f_{3} .
\end{array}\right.
$$

TABLE II: Parameters used in the models

\begin{tabular}{|c|c||c|c|}
\hline Parameter & Value & Parameter & Value \\
\hline$M_{1}$ & $0.06 \mathrm{~kg}$ & $k_{1}$ & $300 \mathrm{~N} / \mathrm{m}$ \\
\hline$M_{2}$ & $0.7 \mathrm{~kg}$ & $k_{2}$ & $9.56 \mathrm{~N} / \mathrm{m}$ \\
\hline$M_{3}$ & $0.03 \mathrm{~kg}$ & $c_{1}$ & $1.2 \mathrm{Ns} / \mathrm{m}$ \\
\hline$P_{\exp }$ & $1.44 \mathrm{~W}$ & $c_{2}$ & $0.1 \mathrm{Ns} / \mathrm{m}$ \\
\hline$r$ & $0.145 \mathrm{~m}$ & $L_{10}$ & $0.45 \mathrm{~m}$ \\
\hline$\omega$ & $17.85 \mathrm{rad} / \mathrm{s}$ & $L_{20}$ & $0 \mathrm{~m}$ \\
\hline$F_{e}$ & $0.56 \mathrm{~N}$ & $F_{c}$ & $1.39 \mathrm{~N}$ \\
\hline
\end{tabular}

Stance phase of Model III:

$$
\left\{\begin{aligned}
\ddot{x}_{1} & =0 \\
M_{2} \ddot{x}_{2} & =-G_{2}-f_{s p 1}+f_{s p 2} \\
M_{3} \ddot{x}_{3} & =-G_{3}-f_{s p 2}+f_{3} .
\end{aligned}\right.
$$

\section{Simulation and Experiment Setup}

Based on the equations of motion of system (8)-(13), this paper implements several simulations to analyze the system. Before stating the further exploration, this subsection introduces the setup of simulation. The simulation environment used in this paper is Matlab and the simulation is running using the variable step integrator ode 45 and absolute and relative error tolerance of $10^{-10} \mathrm{~m}$. For the stability analysis of locomotion behavior, we compare the state variables at apex. If the distance between subsequent values of all state variables is less than $10^{-4}$, this motion behavior is considered as a stable periodic hopping. The parameters used in models are listed in Table II, which are obtained from the Table I or calculated according to (2) to (7), where $c_{1}$ and $c_{2}$ are not physically measured. This is because the $S P_{1}$ exhibits a damping with a nonlinear profile [25] and $c_{2}$ is determined by the energy dissipation inside the motor that cannot be measured. The values of $c_{1}$ and $c_{2}$ in Table II are empirical values obtained in optimization process.

\section{Stability of Models}

Prior to further investigation over the hopping behavior of three models, we explore the models' self-stability in this subsection. A number of simulation trials of hopping are conducted for each model. Every simulation starts at an initial apex point. The relationship between the state variables at $T_{\text {apex }}^{(i)}$ and those at $T_{\text {apex }}^{(i+1)}$ is analyzed by a set of return maps, which are shown in Fig. 5. Although the models are multidimensional, $\theta_{\text {apex }}$ and $x_{2 \text { apex }}$ can represent the basic stability of the systems, thus Fig. 5 shows the return maps of only these two variables.

An important finding from the numerical experiments was that all of three simulation models exhibit "self-stabilizing" behaviors even when we start with a variety of initial conditions. More specifically, each of three models has a specific stable fix point (indicated by a black dot in Fig. 5) which are determined by the design and control parameters, and when we start the simulation with a set of initial conditions near to the fix point, the hopping behaviors always converge to the nominal trajectory. While all three models exhibit the 


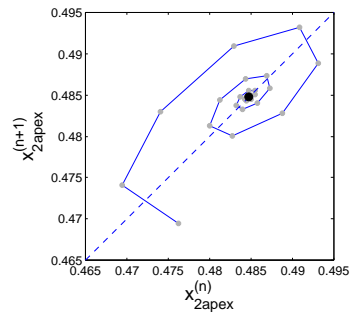

(a) Return map 1 of Model I

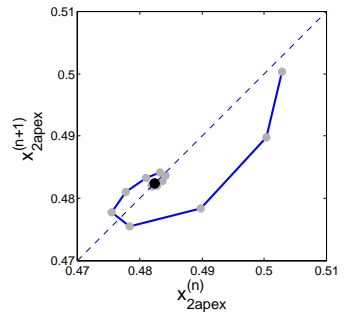

(c) Return map 1 of Model Il

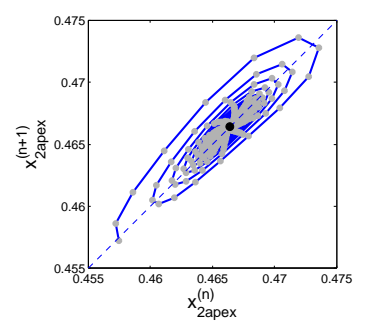

(e) Return map 1 of Model III

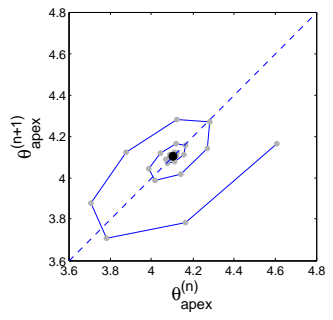

(b) Return map 2 of Model I

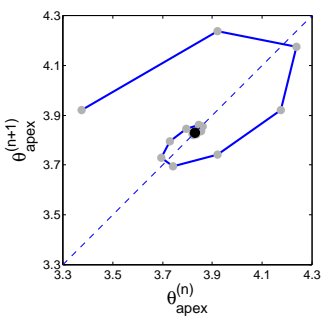

(d) Return map 2 of Model II

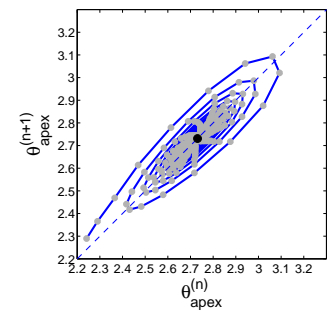

(f) Return map 2 of Model III

Fig. 5: Return maps for three models, i.e., (a) and (b) for Model I, (c) and (d) for Model II, (e) and (f) for Model III. (a), (c) and (e) show the return maps of $x_{2 a p e x}$, which is the value of $x_{2}$ at the apex point. (b), (d) and (f) show the return maps of $\theta_{\text {apex }}$, which is the value of $\theta$ at the apex point. The black solid points in the center stand for the stable fix points.

self-stabilizing behaviors, the convergence speed varies as the Model I and II are usually faster than Model III. In fact, hopping behaviors of the models can be uniquely determined by a set of design parameters, regardless of initial conditions. The models shows a certain 'self-stability'.

Despite the lack of systematic analyses over the system stability, the results in Fig. 5 demonstrate three models are able to exhibit self-stable hopping. Based on this fact, the investigation on the models' hopping behaviors can be discussed. In the rest of this paper, we only analyze self-stable hopping behaviors for further investigations.

\section{E. Analysis of Hopping Behavior}

This subsection shows a feasibility test of the proposed models with respect to our real-world platform. Based on the registered hopping trajectories of the real-world platform (explained in Section II), we conducted a parameter tuning in the proposed models. Fig. 6 shows the time-series trajectories of the input force $f$, power $P$ and all state variables i.e. $x_{1}$, $x_{2}, x_{3}, \dot{x}_{1}, \dot{x}_{2}, \dot{x}_{3}$ in the real-world platform as well as the three models. The experimental results of the robot are shown
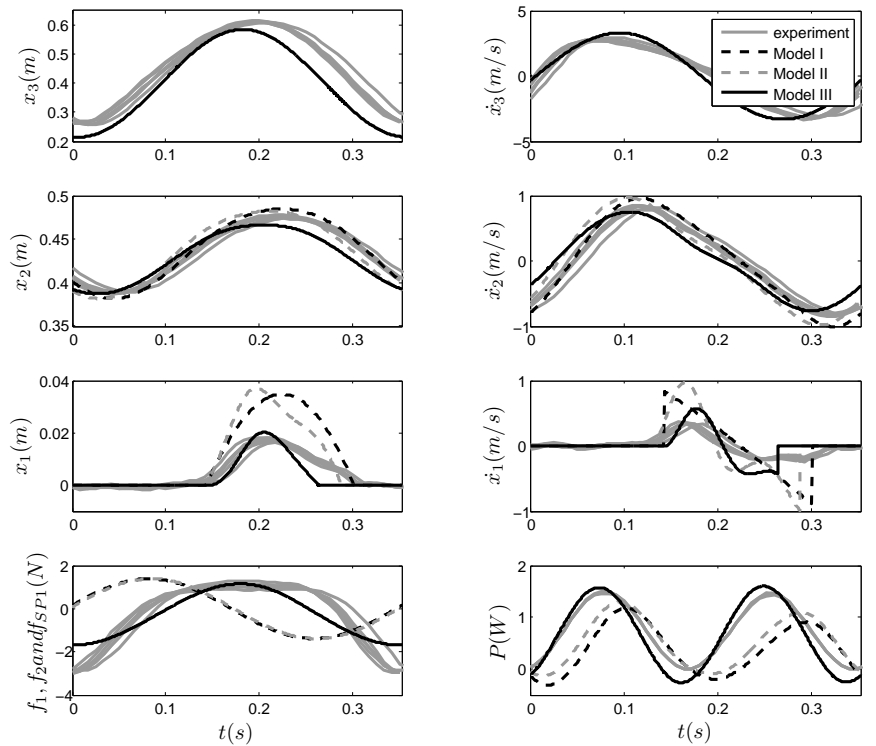

Fig. 6: Trajectory of some variables and performance for one cycle stable behavior of the models in simulation and real robot in experiment. The trajectories of robot during 10 consequent cycles are plotted in one cycle period (gray solid lines). The curves of Model III (black solid lines) are the most close to the curves of robot (gray solid lines).

over 10 steps, while the simulation results show trajectories of one step during the self-stabilizing hopping behaviors at fix points. The identified parameters are shown in Table II.

It is important to note that we calculated the force and power in the simulation as follows. First, although, in Model I and II, $f_{1}$ and $f_{2}$ are plotted as the input forces directly in Fig. 6 , we consider the spring force $f_{s p 2}$ exerted on $M_{2}$ by $S P_{2}$ in Model III. As for the experimental results of the real-world robot, we computed the vertical component of centrifugal force induced by the rotating mass which are estimated by the angular velocity components of motion capture data and the known rotating mass. And second, the input power to each model $P_{1}, P_{2}, P_{3}$ can be obtained by

$$
\left\{\begin{array}{l}
P_{1}=f_{1} \cdot \dot{x}_{2} \\
P_{2}=f_{2} \cdot \dot{x}_{2} \\
P_{3}=f_{3} \cdot \dot{x}_{3} .
\end{array}\right.
$$

In contrast, the input power to the real-world robot was estimated from the consumed electric power measured at the power supply and considering the vertical velocity component of the rotating mass (see more details in Section II).

By observing trajectories of $x_{2}, x_{3}, \dot{x_{2}}$ and $\dot{x_{3}}$, behaviors of all three models can match accurately to those of the realworld robot when the model parameters are properly adjusted. More specifically, all three models are able to hop at a constant period, approximately $0.35 \mathrm{sec}$, which is also the case with the behaviors of the robot. Also the amplitude, offset, and phase delay of the fluctuation in $x_{2}, x_{3}, \dot{x_{2}}$, and $\dot{x_{3}}$ are almost identical (i.e. amplitude and offset of state variables are roughly: $x_{2}^{a m p}=0.04(m), x_{2}^{o f f s e t}=0.43(\mathrm{~m}), \dot{x_{2}}{ }^{a m p}=0.8(\mathrm{~m} / \mathrm{s})$, ${\dot{x_{2}}}^{\text {offset }}=0.0(\mathrm{~m} / \mathrm{s}), x_{3}^{a m p}=0.18(\mathrm{~m}), x_{3}^{\text {off set }}=0.4(\mathrm{~m})$, 
${\dot{x_{3}}}^{a m p}=3(\mathrm{~m} / \mathrm{s})$, and ${\dot{x_{3}}}^{\text {off set }}=0.0(\mathrm{~m} / \mathrm{s}))$.

However, a significant difference is observed in the hopping height $\left(x_{1}\right.$ and $\left.\dot{x_{1}}\right)$, as well as force and power plot in Fig. 6 . Specifically, hopping height of Model I and II is approximately twice as large as those of the robots and Model III (i.e. $0.04(\mathrm{~m})$ versus $0.02(\mathrm{~m})$ ), and the similar large differences are also observed in the trajectories of $\dot{x_{1}}$. Moreover, the trajectories of force and power in Model I and II are delayed by approximately $\frac{\pi}{2}$ compared to those of the robot and Model III, which could never be adjusted over the entire range of model parameters. It is important to note that the phase delays in Model I and II are crucial for the investigation of energy efficiency, because the phase shifts resulted in the negative work of actuation (i.e. the negative power of Model I and II in Fig. 6) which does not reflect the real world experiments.

In summary, these experimental results indicated three findings about the dynamics of the real-world hopping robot. First, from the experimental results in Section III.D, all of three models can represent the self-stabilizing characteristics that were observed in the real-world robot. Second, the basic trajectories of the hopping behaviors (i.e. six state variables) can also be represented by all three models when the model parameters are adjusted. And third, the force generated by the rotating masses and the input power to the system can be only reproduced in the three-mass model (Model III), and the use of one- or two-mass models results in mis-calculation of energy efficiency due to the neglect of rotating-mass dynamics. Given these considerations, we conclude that Model III is the minimal model that explains both the hopping dynamics of the real-world robot as well as its characteristics of energy efficiency.

\section{Simulation Results}

Based on Model III, this section investigates the effect of the control parameters on the vertical hopping behavior. Through this experiment, we explore design parameters of the real-world robot for improving energy efficiency of vertical hopping. Furthermore, we also explore the influences of design parameters with respect to payload and rotating mass. The source of energy loss during the hopping is also analyzed, which provides a strategic plan of design optimization.

\section{A. Effect of Control Parameters}

This subsection first explores the influence of control parameters in Model III, which are the amplitude of input force $F_{e}$ and angular velocity of rotating mass $\omega$. Given a curved beam robot platform in Fig. 1(a), the stable vertical hopping behavior can be found by regulating these two control parameters. The design parameters in Table II are still used for Model III. The periodic stable behaviors are searched in the parameter space of $14.5 \mathrm{rad} / \mathrm{s}$ to $23 \mathrm{rad} / \mathrm{s}$ for $\omega$ and $0.4 N$ to $1 N$ for $F_{e}$.

Fig. 7(a) and 7(b) show the maximum hopping height $(h)$ and the energy dissipation $(E)$ in one cycle hopping. $E$ is calculated by

$$
E=\int_{0}^{T} P d t
$$

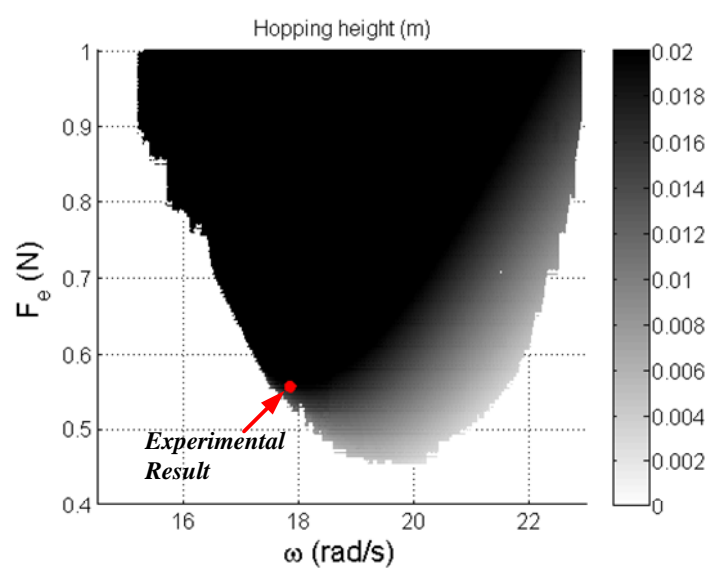

(a)

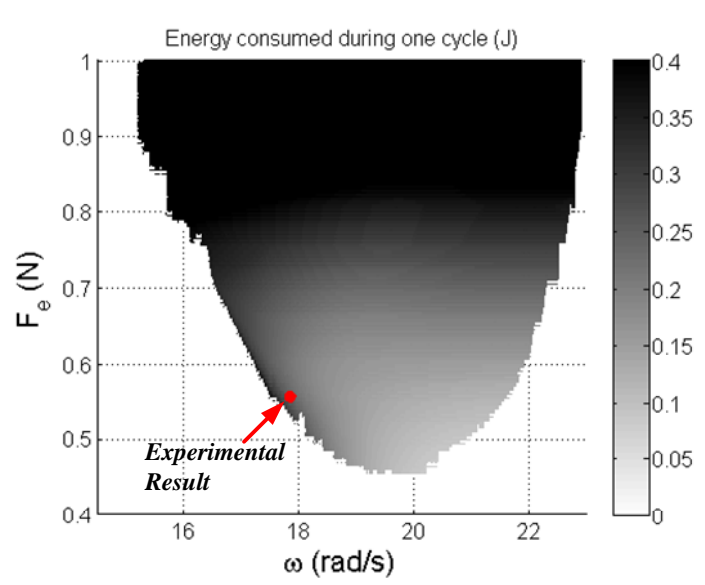

(b)

Fig. 7: (a) The hopping height and (b) the input energy dissipation during one cycle. Based on Model III, a number of simulation are implemented for exploring the stable robot hopping behavior. The area of $F_{e}$ and $\omega$ for stable hopping is shown with gray points. The darker area corresponds the higher hopping height in (a) and more consumed energy in (b). The experimental result proposed in the previous subsection is marked by a red solid dot.

where $T$ is the period of one cycle. Every point in the gray area stands for a set of $F_{e}$ and $\omega$ which can achieve a stable hopping behavior. The gradient in these figures implies the hopping height and input energy during one cycle of hopping. The experimental results in the previous subsection $h$ and $E$ are also presented in the figures, which are marked by red solid points.

There are two main implications that we can observe in these figures. First energy consumption is almost proportional to $F_{e}$, as the lower the $F_{e}$ is, the lower the energy consumption (as shown in Fig. 7(b)). This principle is, however, bounded by the locomotion stability and the resonance frequency, where a close-to resonance frequency (between $\omega=17$ and $18 \mathrm{rad} / \mathrm{s}$ ) increases energy consumption (the resonance frequency of the system when fixed on the ground is approximately $20.7 \mathrm{rad} / \mathrm{s}$ ). And second, both hopping height and energy consumption decrease as we decrease $F_{e}$ and increase $\omega$ (toward the lower 

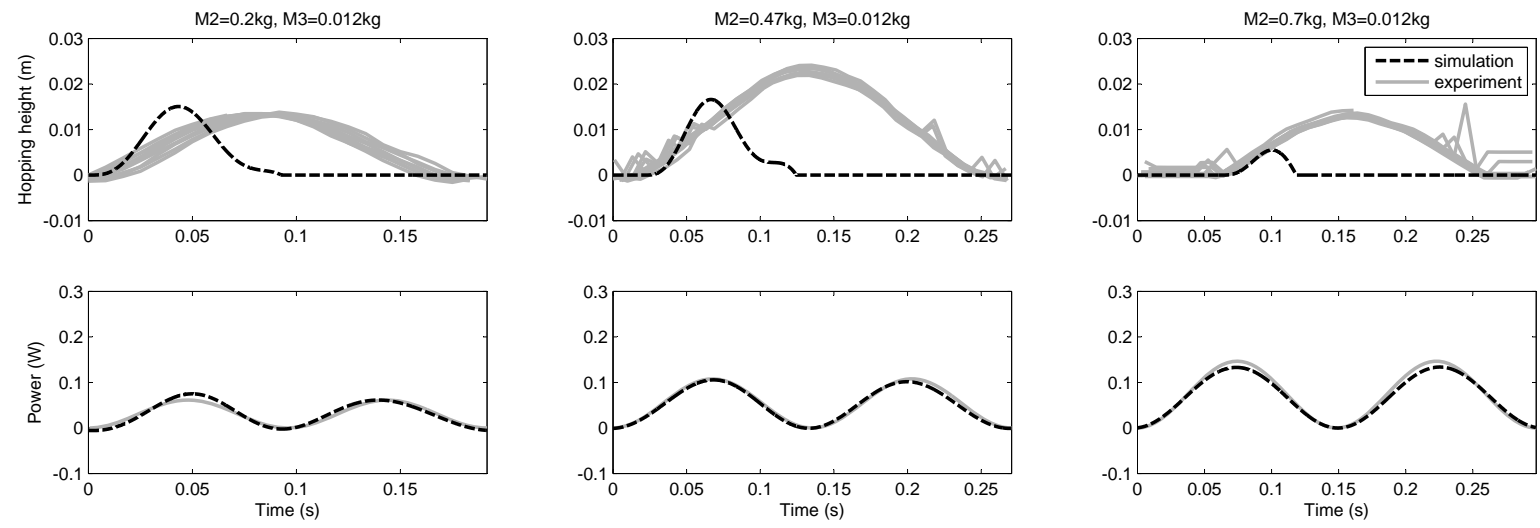

Fig. 8: The trajectories of base mass and input power of the experiment and simulation in one cycle. Three experiments achieve $\mathrm{CoH}$ of 0.23, 0.19 and 0.345, which are considered as high energy efficient compared to the robot in Fig. 1(a) $(\mathrm{CoH}=3.27)$.

right edges in Fig. 7(a) and 7(b).

These results were also observed in the robot hopping experiments. The real-world experiment shown in the previous section is located at the lower left edge in Fig. 7(a) and 7(b). This is mainly because a low $F_{e}$ resulted in no sufficient input energy to the robot for hopping, and $\omega$ needs to be in a middlerange value, since the control frequency has to approach the near-to resonance frequency of the system.

\section{B. Analysis of energy efficiency}

This subsection shows scalability of the proposed approach with respect to the body mass and energy efficiency. By using both the real-world robot and the simulation model, we analyze how $\mathrm{CoH}$ is related to body mass, hopping height and energy expenditure during stable hopping, and identify the underlying mechanisms for the scalability.

In the first set of experiments, we explored how the realworld robot platform exhibits stable hopping under different body mass. In these experiments, we made use of the same robot shown in Fig. 1(a) and Table I, and we explored $\mathrm{CoH}$ when different masses are added to the top of the real-world robot (i.e. three different variations of $M_{2}=0.2,0.47$, and $0.7(k g)$ ). In order to minimize $\mathrm{CoH}$, we heuristically searched the input power to the motor such that the robot exhibits the lowest $\mathrm{CoH}$ while compromising with hopping height. As a result of the optimization, we found the best performance can be achieved when the rotating mass is $0.012 \mathrm{~kg}$ and the input power is $0.15 \mathrm{~W}$. The experimental results are shown in Fig. 8. In general, the figure shows that the proposed approach can be used for stable hopping with different payloads while $\mathrm{CoH}$ to be maintained between 0.19 and 0.35 , which is significantly lower than the experiment in Fig. 6. Given these experimental results, we also investigated Model III to figure out whether a similar characteristics can be observed. In the numerical simulation, we employed the values of the frequency $\omega$, payload $M_{2}$ and rotating mass $M_{3}$ used in the experiment while the values of rest parameters came from Table II. We conducted an optimization over the parameters of power input $F_{e}$, and damping coefficients $c_{1}$ and $c_{2}$ in the simulation to make the trajectories of input power close to the experimental results. We found Model III is capable of exhibiting similar stable hopping with respect to maximum hopping height and efficiency under different payloads (see dashed lines in Fig. 8). While the input power in the simulation is close the one in the real-world experiment, the hopping height differs. This is because of a number of assumptions inside Model III, e.g. the curved beam is considered as a linear spring in Model III while it exhibits a nonlinear profile. Although there exists a difference from the real-world experiment, Model III as a minimalistic model is demonstrated to be able to characterize the robot hopping and show similar performance with different payloads.

Although we were not able to increase body and rotating masses due to the hardware limitations in the curved beam and electric motor, the following analysis shows further details on the scalability of the proposed approach by investigating Model III. Here we applied different $M_{2}$ and $M_{3}$, while maintaining the rest of parameters unchanged as shown in Table II. To analyze the relationship between $\mathrm{CoH}$ and body masses, the open parameter (i.e. control parameters $F_{e}$ ) was adjusted for stable vertical hopping with the lowest $\mathrm{CoH}$, and the results are plotted in Fig. 9(a) together with the hopping height and energy consumption.

By observing the plots of different payload in Fig. 9(a), there is a minimum $\mathrm{CoH}$ that requires an intermediate $M_{3}$. Small $M_{3}$ leads to a significant increase of $\mathrm{CoH}$, while excessively large $M_{3}$ increases $C o H$ slowly. Fig. 9(b) and 9(c) also show that $E$ and $h$ both rise with $M_{3}$ and a larger $M_{2}$ results in a gentler rising slope. Moreover, the plots of $\mathrm{CoH}$ with the same $\mathrm{M}_{3}$ decrease when $M_{2}$ becomes larger, which means larger payload is helpful to gain a lower $\mathrm{CoH}$.

In order to further analyze the source of energy consumption, the energy consumed in the damping of spring $S P_{1}$ and $S P_{2}$, and collision in one cycle stable behavior are shown in Fig. 10. Here only the results of the system with $M_{2}=0.7 \mathrm{~kg}$ are shown. As shown in the figure, the energy consumption in collision for each system is small compared to the spring damping. It is reasonable as a small base mass is used to considerably reduce the energy loss in collision. Interestingly, the energy consumed in the damping of spring $S_{2}$ falls with $M_{3}$, while the one for the spring $S P_{1}$ rises. This is because the 


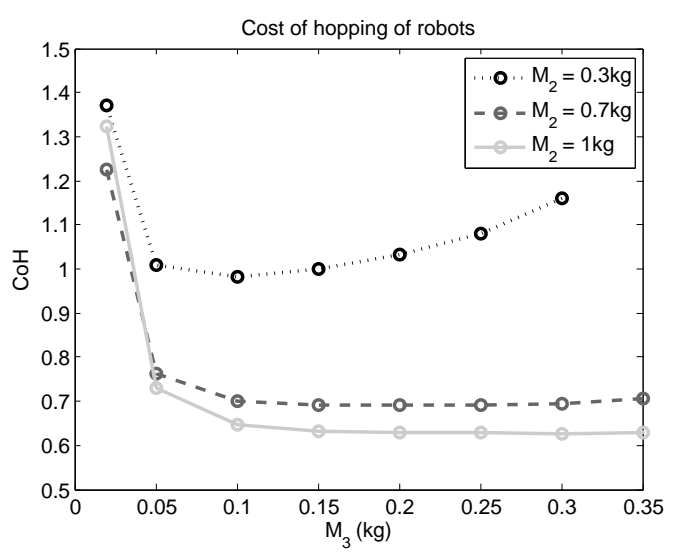

(a)

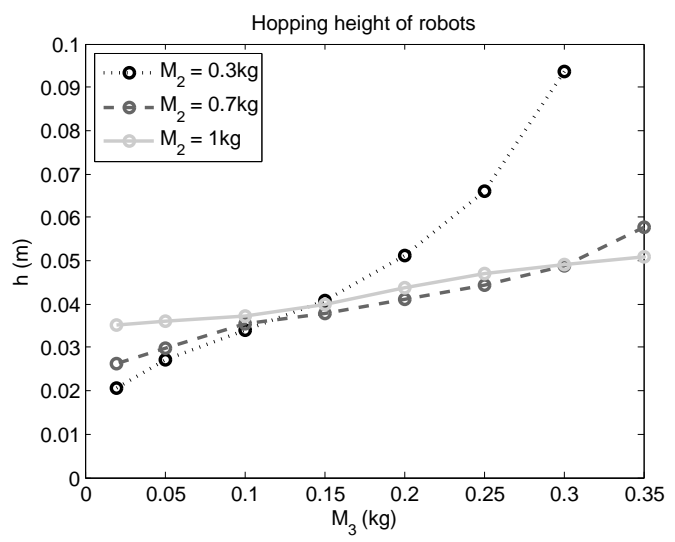

(b)

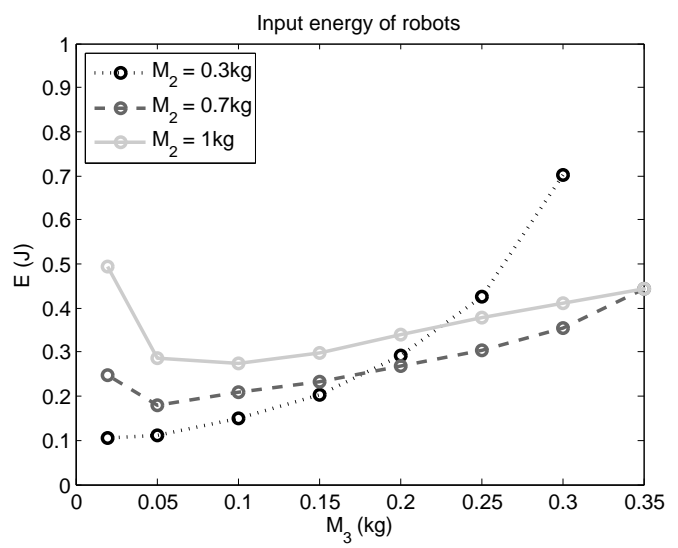

(c)

Fig. 9: (a) Optimal $\mathrm{CoH}$ of periodic hopping behaviors with certain design parameters. For one system, the optimal $\mathrm{CoH}$ is found by regulating control parameters $\omega$ and $F_{e}$, and plotted as a point. These systems have different $M_{2}$ and $M_{3}$. The optimal $\mathrm{CoH}$ points of the systems with the same $M_{2}$ are connected and plotted using one line style. (b) and (c) show the energy consumption in one behavioral cycle $E$ and hopping height $h$ of the systems in (a).

amplitude of compression of the spring $S P_{1}$ is strengthened as $M_{3}$ rises, while the one for the spring $S P_{2}$ falls. It shows that the increase of rotating mass weakens the relative vibration

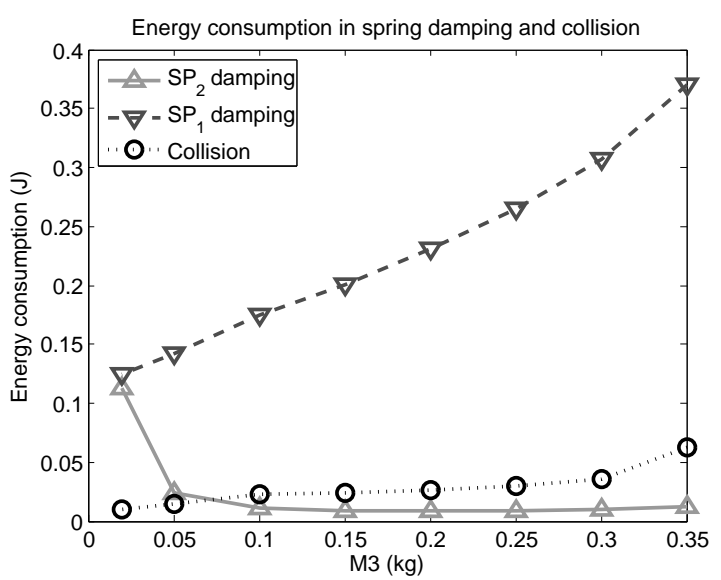

Fig. 10: The energy dissipation of systems in the damping of spring $S P_{1}$ and $S P_{2}$, and collision. The systems with $M_{2}=$ $0.7 \mathrm{~kg}$ shown in Fig. 9 is analyzed.

between the rotating mass and payload, which implies the radius of rotating mass is shortened. Therefore, the value of $M_{3}$ has a upper bound due to this physical constraints in the experiment. Above all, the amount of the energy dissipation of system is mainly determined by the damping of the spring $S P_{1}$ (i.e. the damping of elastic body). Thus the damping coefficient $c_{1}$ should be first considered to reduce during the design of robot.

\section{CONClusion}

This paper presented a series of experiments, modeling and design analysis of a curved beam robot that we developed. By quantifying the energy efficiency of hopping behavior, i.e. Cost of Hopping, we evaluated our robot as compared to a few different systems including human and the other robots. Through the system identification with a few different minimalistic models, we found that our robot has an intrinsic stability in hopping behaviors while keeping the energy dissipation very low. The self-stabilizing characteristics is particularly important in our framework because it allows us to systematically analyze the design space which is the main driving force of hopping behaviors. Through the numerical simulation and realworld experiments of various design and control parameters, we further identified the strategy to optimize our robots for better hopping performances. In principle, our approach is fairly scalable with respect to the size and payload, while the motor control (i.e. rotating mass, the rotational radius and frequency) has to be adjusted accordingly. Furthermore, we also identified that the damping of the leg spring is the main source of energy dissipation because the comparatively small mass on the foot does not cause significant energy loss at impact. Therefore the next important step of this research is to improve the damping characteristics in the body structure of the robot.

In general, energy efficiency of robotic systems is not a trivial problem to theoretically estimate because energy consumption of real-world systems involves many complex physical processes for modelling and simulation such as 
friction, impact, damping in nonlinear dynamics as well as material properties and chemical reactions for energy conversion in batteries, for example. Nevertheless, we hope that the minimalistic approach as we presented in this paper will help understanding a basic strategy to fundamentally improve energy efficiency of robotic systems. In the future, it is also expected to investigate how the basic strategy can be extended to more complex ones, including those which carry more spring-mass-damper components.

\section{REFERENCES}

[1] F. Iida, M. Reis, N. Maheshwari, K. Gunura, and S. Hauser, "Legged robot locomotion based on free vibration," in Proc. IEEE Int. Workshop on Adv. Motion Control, March 2012, pp. 1-6.

[2] X. Yu and F. Iida, "Minimalistic models of an energy efficient vertical hopping robot," in Proc. IEEE Int. Conf. Robot. Autom., May 2013, pp. $7-12$.

[3] B. Ugurlu and A. Kawamura, "Zmp-based online jumping pattern generation for a one-legged robot," IEEE Trans. Ind. Electron., vol. 57, no. 5, pp. 1701-1709, May 2010.

[4] K. Loffler, M. Gienger, F. Pfeiffer, and H. Ulbrich, "Sensors and control concept of a biped robot," IEEE Trans. Ind. Electron., vol. 51, no. 5, pp. 972-980, Oct 2004.

[5] K. Erbatur and O. Kurt, "Natural zmp trajectories for biped robot reference generation," IEEE Trans. Ind. Electron., vol. 56, no. 3, pp. 835-845, March 2009.

[6] C. Fu and K. Chen, "Gait synthesis and sensory control of stair climbing for a humanoid robot," IEEE Trans. Ind. Electron., vol. 55, no. 5, pp. 2111-2120, May 2008.

[7] C.-F. Juang, Y.-C. Chang, and C.-M. Hsiao, "Evolving gaits of a hexapod robot by recurrent neural networks with symbiotic species-based particle swarm optimization," IEEE Trans. Ind. Electron., vol. 58, no. 7, pp. 3110-3119, July 2011.

[8] T. Von Karman and G. Gabrielli, "What price speed? specific power required for propulsion of vehicles," Mech. Eng., vol. 72, pp. 775-781, 1950.

[9] V. A. Tucker, "The energetic cost of moving about," Am. Sci., vol. 63, no. 4, pp. 413-419, 1975.

[10] M. H. Dickinson, C. T. Farley, R. J. Full, M. A. R. Koehl, R. Kram, and S. Lehman, "How animals move: An integrative view," Science, vol. 288, no. 5463, pp. 100-106, 2000.

[11] R. Alexander, "Three uses for springs in legged locomotion," Int. J. Robot. Res., vol. 9, no. 2, pp. 53-61, April 1990.

[12] A. D. Kuo, "Choosing your steps carefully," IEEE Robot. Automat. Mag., vol. 14 , no. 2 , pp. 18-29, june 2007

[13] T. McGeer, "Passive dynamic walking," Int. J. Robot. Res., vol. 9, no. 2 , pp. 62-82, April 1990.

[14] S. Collins, A. Ruina, R. Tedrake, and M. Wisse, "Efficient bipedal robots based on passive-dynamic walkers," Science, vol. 307, no. 5712, pp. 1082-1085, February 2005.

[15] D. Owaki, M. Koyama, S. Yamaguchi, S. Kubo, and A. Ishiguro, "A 2-d passive-dynamic-running biped with elastic elements," IEEE Trans. Robot., vol. 27, no. 1, pp. 156-162, 2011.

[16] M. H. Raibert, Legged robots that balance. Cambridge, MA: The MIT Press, 1985

[17] A. Chatterjee, R. Pratap, C. Reddy, and A. Ruina, "Persistent passive hopping and juggling is possible even with plastic collisions," Int. J. Robot. Res., vol. 21, no. 7, pp. 621-634, July 2002.

[18] M. Hutter, C. D. Remy, M. A. Hoepflinger, and R. Siegwart, "Efficient and versatile locomotion with highly compliant legs," IEEE/ASME Trans. Mechatron., vol. 18, no. 2, pp. 449-458, 2013.

[19] R. Blickhan, "The spring-mass model for running and hopping," $J$. Biomech, vol. 22, no. 11-12, pp. 1217-1227, 1989.

[20] T. A. McMahon and G. C. Cheng, "The mechanics of running: How does stiffness couple with speed?" J. Biomech, vol. 23, Supplement 1, pp. 65-78, 1990.

[21] A. Seyfarth, H. Geyer, M. Gnther, and R. Blickhan, "A movement criterion for running," J. Biomech, vol. 35, no. 5, pp. 649-655, May 2002.

[22] H. Geyer, A. Seyfarth, and R. Blickhan, "Spring-mass running: simple approximate solution and application to gait stability," J. Theor. Biol., vol. 232 , no. 3 , pp. $315-328,2005$.
[23] M. Ahmadi and M. Buehler, "The arl monopod ii running robot: control and energetics," in Proc. IEEE Int. Conf. Robot. Autom., vol. 3, 1999, pp. 1689-1694.

[24] G. Zeglin and B. Brown, "Control of a bow leg hopping robot," in Proc. IEEE Int. Conf. Robot. Autom., vol. 1, May 1998, pp. 793-798.

[25] M. Reis and F. Iida, "An energy efficient hopping robot based on free vibration of a curved beam," IEEE/ASME Trans. Mechatron., to be published.

[26] M. Reis, X. Yu, N. Maheshwari, and F. Iida, "Morphological computation of multi-gaited robot locomotion based on free vibration," Artif. Life, vol. 19, no. 1, pp. 97-114, 2013.

[27] N. Maheshwari, X. Yu, M. Reis, and F. Iida, "Exploiting free vibration of an elastic beam for stable running locomotion," in Proc. IEEE/ASME Int. Conf. Adv. Intell. Mechatron., July 2012, pp. 27-32.

[28] A. M. Grabowski and H. M. Herr, "Leg exoskeleton reduces the metabolic cost of human hopping," J. Appl. Physiol., vol. 107, no. 3, pp. 670-678, 2009.

[29] M. Kovac, M. Fuchs, A. Guignard, J. C. Zufferey, and D. Floreano, "A miniature $7 \mathrm{~g}$ jumping robot," in Proc. IEEE Int. Conf. Robot. Autom., 2008, pp. 373-378.

[30] M. Ahmadi and M. Buehler, "Stable control of a simulated onelegged running robot with hip and leg compliance," IEEE Trans. Robot. Automat., vol. 13, no. 1, pp. 96-104, February 1997.

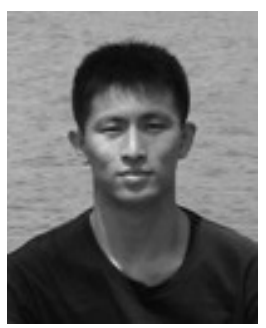

Xiaoxiang Yu received the B.Eng. degree in automation engineering from the Dalian University of Technology, Dalian, China, in 2008 and the M.Sc. degree in control theory and control engineering from the Shanghai Jiao Tong University, Shanghai, China, in 2011. He is currently working toward the Ph.D. degree at the Bio-Inspired Laboratory, ETH Zurich, Switzerland.

His research interests include dynamic legged locomotion and dynamics.

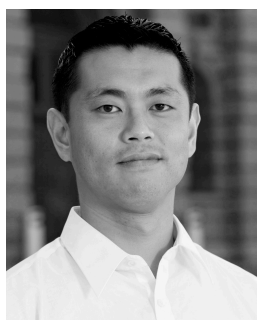

Fumiya Iida (M'11) received the Bachelors and Masters degrees in mechanical engineering from the Tokyo University of Science, Tokyo, Japan, in 1999, and the Dr. sc. nat. degree in informatics from the University of Zurich, Zurich, Switzerland, in 2006. In 2004 and 2005, he was also engaged in biomechanics research of human locomotion at the Locomotion Laboratory, University of Jena, Germany. From 2006 to 2009, he worked as a Postdoctoral Associate at the Computer Science and Artificial Intelligence Laboratory, Massachusetts Institute of Technology. He has been an assistant professor and the director of the Bio-Inspired Robotics Laboratory at ETH Zurich since August 2009. His research interests include biologically inspired robotics, embodied artificial intelligence, and biomechanics, where he was involved in a number of research projects related to dynamic legged locomotion, navigation of autonomous robots, and human-machine interactions. He was awarded the Fellowship for Prospective Researchers from the Swiss National Science Foundation in 2006 , and the Swiss National Science Foundation Professorship in 2009. 